\title{
Risk factors for brain metastases in patients with non-small cell lung cancer: a meta-analysis of $\mathbf{4 3}$ studies
}

\author{
Shanshan Chen ${ }^{1 \#}$, Xin Hua ${ }^{1 \#}$, Jinfang Jia ${ }^{1}$, Ying $\mathrm{Wu}^{2}$, Shuzhen Wei ${ }^{2}$, Lu Xu ${ }^{1,2}$, Shuhua Han ${ }^{1,2}$, \\ Hongming Zhang ${ }^{3}$, Xiaoli Zhu ${ }^{1,2}$
}

${ }^{1}$ School of Medicine, Southeast University, Nanjing, China; ${ }^{2}$ Department of Respiratory Medicine, Zhongda Hospital of Southeast University, Nanjing, China; ${ }^{3}$ Department of Respiratory Medicine, Yancheng Third People's Hospital, the Affiliated Yancheng Hospital of Southeast University Medical College, Yancheng, China

Contributions: (I) Conception and design: H Zhang, X Zhu; (II) Administrative support: S Chen, X Hua; (III) Provision of study materials or patients: S Han, J Jia, L Xu; (IV) Collection and assembly of data: S Chen, X Hua; (V) Data analysis and interpretation: S Wei, Y Wu; (VI) Manuscript writing: All authors; (VII) Final approval of manuscript: All authors.

\#These authors contributed equally to this work.

Correspondence to: Hongming Zhang. Department of Respiratory Medicine, Yancheng Third People's Hospital, the Affiliated Yancheng Hospital of Southeast University Medical College, Yancheng 224000, China. Email: hmzhang149252@163.com; Xiaoli Zhu. School of Medicine, Southeast University, Nanjing 210009, China. Email: zhuxiaolipaper@126.com.

Background: Lung cancer is a leading cause of cancer-related mortality worldwide. The purpose of our meta-analysis was to assess the risk factors for brain metastases (BM) in patients with non-small cell lung cancer (NSCLC).

Methods: Multiple databases, including PubMed, EMBASE, Cochrane Library, China National Knowledge Infrastructure (CNKI), and Wanfang, were systematically searched to recruit relevant studies investigating the risk factors for BM in NSCLC patients. The Newcastle-Ottawa Scale was used to evaluate literature quality, and the meta-analysis was performed using the Review Manager 5.3. Evidence quality evaluation was carried out according to the Grading of Recommendation Assessment, Development and Evaluation (GRADE) standard. The estimated odds ratio (OR) and 95\% confidence intervals (CIs) were set as effect measures. Funnel plots and sensitivity analyses were used to assess publication bias and the robustness and reliability of the combined results, respectively.

Results: A total of 43 studies with 11,415 participants were included in this meta-analysis. The results indicated that the following factors were significantly associated with an increased risk of BM in NSCLC patients $(\mathrm{P}<0.05)$ : (I) gender (female) $(\mathrm{OR}=1.32,95 \% \mathrm{CI}: 1.17-1.49, \mathrm{P}<0.00001)$; (II) adenocarcinoma (OR $=2.34,95 \% \mathrm{CI}: 1.76-3.11, \mathrm{P}<0.00001)$ or non-squamous cell carcinoma $(\mathrm{OR}=0.63,95 \% \mathrm{CI}: 0.42-0.94$, $\mathrm{P}=0.02)$; (III) advanced tumor stage ( $\mathrm{OR}=1.48,95 \% \mathrm{CI}: 1.01-2.17, \mathrm{P}=0.04)$; (IV) node stage $(\mathrm{OR}=2.19$, 95\% CI: 1.39-3.45, P=0.0007); (V) lymphatic metastasis (OR =2.43, 95\% CI: 1.76-3.36, $\mathrm{P}<0.00001)$; (VI) epidermal growth factor receptor (EGFR) gene mutation ( $\mathrm{OR}=1.88,95 \% \mathrm{CI}: 1.26-2.80, \mathrm{P}=0.002)$; (VII) kirsten rat sarcoma viral oncogene (KRAS) gene mutation (OR =2.99, 95\% CI: 1.82-4.91, $\mathrm{P}<0.00001)$; (VIII) higher levels of carcinoembryonic antigen $(\mathrm{P}<0.00001)$, carbohydrate antigen $199(\mathrm{P}<0.0001)$, cytokeratin-19 fragment $(\mathrm{P}=0.04)$, neuron-specific enolase $(\mathrm{P}<0.00001)$, and carbohydrate antigen $125(\mathrm{P}=0.0005)$.

Conclusions: This meta-analysis demonstrated that NSCLC patients with BM have more aggressive clinical features.

Keywords: Non-small cell lung cancer (NSCLC); brain metastases (BM); risk factors; meta-analysis; prognosis

Submitted Aug 27, 2020. Accepted for publication Dec 31, 2020.

doi: 10.21037/apm-20-1722

View this article at: http://dx.doi.org/10.21037/apm-20-1722 


\section{Introduction}

Lung cancer is one of the most common malignant tumors worldwide (1). Non-small cell cancer (NSCLC) is the prevailing histological subtype of lung cancer, accounting for approximately $80-85 \%$ (2). Given that its early clinical symptoms are not typical, NSCLC diagnosis is usually based on tumor markers, imaging, and histopathological characteristics. It is estimated that approximately $40 \%$ of NSCLC patients present with concomitant metastatic disease at initial diagnosis $(3,4)$, most commonly of the brain, bone, liver, etc. Despite advancements in therapy (1), the prognosis for patients with advanced lung cancer is not good, especially for patients combined with brain metastases (BM) (5).

Previous studies have shown that BM is a key cause of morbidity and mortality in cancer and that approximately $20-40 \%$ of NSCLC patients will develop BM (6). The median survival period of NSCLC patients with BM is only about 3-6 months (7). Although some targeted methods can play a role in controlling the intracranial metastasis of tumors, few drugs can effectively cross the blood-brain barrier (8). At present, radiation therapy and surgical intervention are the most effective therapeutic options for BM; however, these two treatments may significantly impact the quality of life of patients (9). A meta-analysis of the risk factors for the occurrence of BM in NSCLC has not yet been performed. Therefore, there is a pressing need for greater assessment of the risk factors associated with BM in NSCLC patients, which may allow for prevention and earlier treatment of $\mathrm{BM}$ and help patients achieve prolonged survival. The purpose of our meta-analysis was to summarize the risk factors and clinical characteristics of NSCLC patients with BM.

We present the following article in accordance with the PRISMA reporting checklist (available at http://dx.doi. org/10.21037/apm-20-1722).

\section{Methods}

\section{Search strategy}

Various databases, including PubMed, EMBASE, Cochrane Library, China National Knowledge Infrastructure (CNKI), and WanFang, were systematically searched from the date of inception of the database to February 2020, without language, publication, or time restrictions. The search terms included "brain metastases", "nervous metastases" or "cerebral metastases" and "non-small cell cancer", "lung cancer" or "malignant lung disease". Finally, we reviewed the references of the relevant studies to identify potentially related articles.

\section{Eligibility/exclusion criteria}

\section{Inclusion criteria}

(I) Cross-sectional, case-control, and cohort studies assessing the risk factors for NSCLC patients with BM.

(II) Studies with clear and unified diagnostic criteria for NSCLC and BM, including histologically or cytologically confirmed NSCLC, and imaging findings, including computed tomography (CT), magnetic resonance imaging (MRI), or positron emission tomography (PET) confirmed BM.

(III) Odds ratio (OR), the hazard ratio (HR), relative risk (RR), or weighted mean difference (WMD) and 95\% confidence intervals (95\% CIs) for BM development and clinicopathological factors could be obtained from multivariate analysis or could be calculated based on relevant data.

(IV) The number of cancers with $\mathrm{BM}$ in the article was more than 20.

\section{Exclusion criteria}

(I) Studies based on overlapping patients.

(II) Meta-analyses, reviews, case reports, or reports based on expert experience.

(III) No effective data like ominous, poor quality, and repeated documents could be extracted.

\section{Data extraction and assessment of study quality}

Two reviewers (Chen and Hua) independently extracted the data from all included studies, and any disagreements were resolved by discussion with the third reviewer (Zhang). The following data were retrieved from the studies: (I) basic characteristics including the first author's name, year of publication, country, characteristics of the study population (e.g., gender, age, number), and study design; (II) clinical characteristics, including age, gender, smoking history, treatment history, pathological type, tumor $(\mathrm{T})$ stage, node (N) stage, lymphatic metastasis, distant metastasis (except outside the brain), epidermal growth factor receptor (EGFR) gene mutation, Kirsten rat sarcoma viral oncogene (KRAS) gene mutation, and ECOG scale; (III) clinical laboratory parameters, including the levels of carcinoembryonic antigen (CEA), carbohydrate antigen 199 (CA199), cytokeratin-19 fragment (CYFRA21-1), neuron-specific 


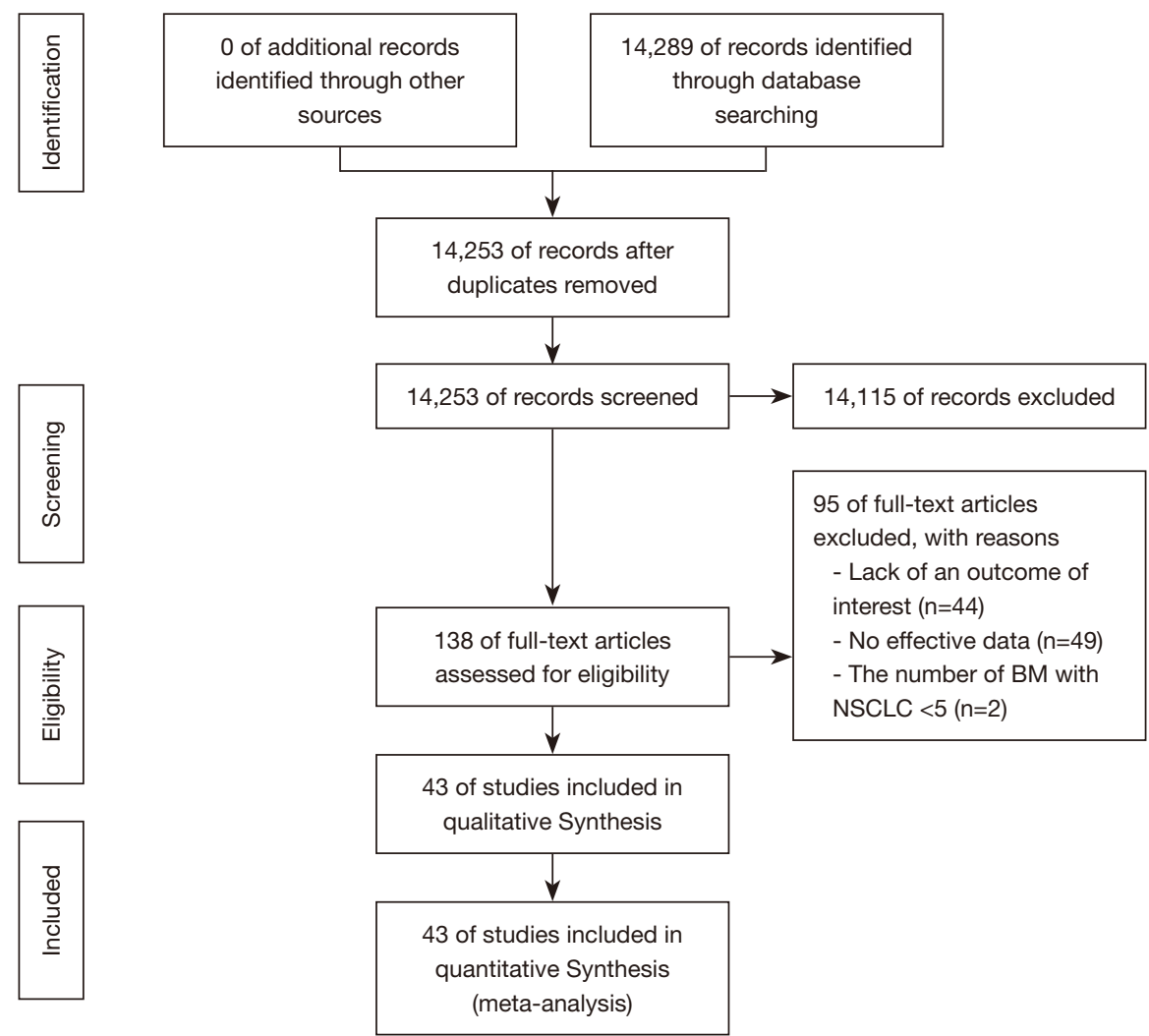

Figure 1 Selection of the included studies.

enolase (NSE), and carbohydrate antigen 125 (CA125).

The included studies' quality was assessed using the Newcastle-Ottawa Scale (NOS) (10); scores of 5-9 were considered fair, while scores of $1-4$ indicated a high risk of bias. The results of this meta-analysis were evaluated using the GRADE profiler, and degradation was assessed in terms of evidence quality, including the risk of bias, inconsistency, indirectness, imprecision, and publication bias. Simultaneously, upgradation was expressed by large effect, plausible confounding, and dose-response gradient (11).

\section{Statistical analysis}

Data in our meta-analysis were analyzed using Review Manager (Version 5.3. Copenhagen: The Nordic Cochrane Centre, The Cochrane Collaboration, 2014) software. The estimated OR and WMD were used to evaluate the affiliation between the incidence of BM and the clinicopathological features of NSCLC patients. All statistic values were reported with $95 \%$ CIs, and the twosided $\mathrm{P}$ value threshold for statistical significance was set at
0.05. The Chi-square test and the $\mathrm{I}^{2}$ statistic were used to evaluate heterogeneity among studies. Specifically, $\mathrm{I}^{2}>50 \%$ and $\mathrm{P}<0.05$ for the Chi-square test suggested significant heterogeneity among the included studies. When the homogeneity hypothesis was not rejected, a fixed-effects model was used; otherwise, a random-effects model was used to estimate the OR and 95\% CI (12).

To investigate the effects of individual studies on the overall results, we also performed a sensitivity analysis by successively excluding each study. Finally, a funnel plot was used to assess potential publication bias. Considering that a small number of included studies may result in publication bias, Egger's and Begg's Tests and funnel plots were generated for indexes with more than 10 relevant studies (13).

\section{Results}

\section{Baseline study characteristics and quality assessment}

We identified 14,289 studies in our initial literature search, as shown in Figure 1. Thirty-six studies were excluded due to duplication. After reviewing the titles and abstracts, 
we identified 138 potentially eligible studies for full-text assessment. A further 95 studies were excluded because they either lacked an outcome of interest or had no compelling information and control groups. Ultimately, 43 studies met our selection criteria and were included in the final analysis. All studies' characteristics and demographic data are presented in Table 1. The retrieved studies were published between 2004 and February 2020, and a total of 11,415 patients were involved. Of the 43 included studies, 26 were cohort studies, and the remaining 17 were case-control studies. Since all of the included studies were either cohort or case-control studies, the NOS was used for quality assessment, and the results showed that all included studies were of fair quality (Table 1).

\section{A meta-analysis of clinical characteristics of patients}

Our meta-analysis (Figure 2) suggested that the prevalence of $\mathrm{BM}$ was significantly higher among female patients $(\mathrm{OR}=1.32$, 95\% CI: $1.17-1.49, \mathrm{P}<0.00001)$ (Figure $2 B)$. However, patients that were younger than 60 years old $(\mathrm{OR}=1.12,95 \%$ CI: $0.97-1.29, \mathrm{P}=0.13$ ) (Figure $2 A)$, had a history of smoking ( $\mathrm{OR}=1.53,95 \% \mathrm{CI}$ : $1.00-2.34, \mathrm{P}=0.05)$ (Figure $2 C$ ), and treatment history $(\mathrm{OR}=0.77,95 \% \mathrm{CI}$ : 0.54-1.11, $\mathrm{P}=0.16$ ) (Figure 2D) did not show significant differences between the NSCLC with BM group and the sample NSCLC group. Obvious heterogeneity was observed in age $\left(\mathrm{I}^{2}=90 \%, \mathrm{P}<0.00001\right)$ and smoking history $\left(\mathrm{I}^{2}=59 \%, \mathrm{P}=0.02\right)$, and thus, a random-effects model was utilized. A fixed-effects model was also used for the other indexes, as there was no obvious heterogeneity in the above studies.

\section{A meta-analysis of tumor-related indexes}

Our meta-analysis (Figure 3) indicated that adenocarcinoma $(\mathrm{OR}=2.34,95 \% \mathrm{CI}: 1.76-3.11, \mathrm{P}<0.00001)$ (Figure $3 A$ ) was a risk factor for BM in NSCLC patients. Conversely, squamous carcinoma was found to be a protective factor $(\mathrm{OR}=0.63,95 \% \mathrm{CI}: 0.42-0.94, \mathrm{P}=0.02)$ (Figure $3 B$ ). Meanwhile, the prevalence of BM was significantly higher among patients with higher $\mathrm{T}$ stage $(\mathrm{OR}=1.48,95 \% \mathrm{CI}$ : $1.01-2.17, \mathrm{P}=0.04)$ (Figure $3 C)$, higher $\mathrm{N}$ stage $(\mathrm{OR}=2.19$, 95\% CI: $1.39-3.45, \mathrm{P}=0.0007$ ) (Figure $3 D$ ), the number of lymphatic metastasis larger than six $(\mathrm{OR}=2.43,95 \% \mathrm{CI}$ : 1.76-3.36, $\mathrm{P}<0.00001$ ) (Figure 3E), EGFR gene mutation ( $\mathrm{OR}=1.88,95 \% \mathrm{CI}: 1.26-2.80, \mathrm{P}=0.002)$ (Figure $3 G$ ), and KRAS gene mutation $(\mathrm{OR}=2.99,95 \% \mathrm{CI}: 1.82-4.91$,
$\mathrm{P}<0.00001$ ) (Figure $3 H$ ). In contrast, patients with other distant metastases (OR $=0.81,95 \%$ CI: $0.29-2.33, \mathrm{P}=0.7$ ) (Figure $3 F)$ and ECOG scale (OR $=1.15$, 95\% CI: 0.78-1.70, $\mathrm{P}=0.47$ ) (Figure 3I) did not show significant differences between the two groups. Since there was no obvious sample heterogeneity in the above studies, a fixed-effects model was utilized, while a random-effects model was used for studies with obvious heterogeneity.

\section{A meta-analysis of clinical laboratory parameters}

The results of our meta-analysis showed that NSCLC patients with BM had higher levels of CEA (WMD $=10.94$, 95\% CI: 7.47-14.40, $\mathrm{P}<0.00001$ ) (Figure 4A), CA199 (WMD $=20.23$, 95\% CI: 12.20-28.26, $\mathrm{P}<0.0001$ ) (Figure 4B), CYFRA211 (WMD $=1.78,95 \%$ CI: 0.04-3.51, P=0.04) (Figure 4C), NSE (WMD =9.66, 95\% CI: 6.18-13.14, $\mathrm{P}<0.00001$ ) (Figure 4D), and CA125 (WMD $=22.39,95 \% \mathrm{CI}$ : 9.79-34.98, $\mathrm{P}=0.0005$ ) (Figure 4E). Obvious heterogeneity was observed among these five indexes $\left(\mathrm{I}^{2}>50 \%\right.$; $\left.\mathrm{P}<0.05\right)$, and thus, a random-effects model was utilized.

\section{Sensitivity analysis and risk of bias}

The NOS Quality Assessment (Table 1) and GRADE evaluation (Figures 5,6) indicated that the included studies were of acceptable quality. A sensitivity analysis was conducted to evaluate each included study's influence; the results showed that heterogeneity and the pooled ORs or WMDs of BM were not significantly altered by any single study, indicating that our conclusions were relatively reliable. Funnel plots were generated for the indexes and are shown in Figures S1-S3. Egger's and Begg's tests are shown in Figures S4,S5.

\section{Discussion}

Our meta-analysis of 43 studies involving 11,415 participants assessed the risk factors and prognosis of $\mathrm{BM}$ in NSCLC patients. Our findings may be important in the prevention and evaluation of NSCLC patients with $\mathrm{BM}$. The results were divided into four categories: clinical characteristics, tumor-related indexes, clinical laboratory parameters, and survival rates of patients.

By examining all relevant studies, we found that gender (female), adenocarcinoma or non-squamous cell carcinoma, advanced tumor stage, node stage, lymphatic metastasis, EGFR gene mutation, KRAS gene mutation, as well as 
Table 1 Basic characteristics of the retrieved studies.

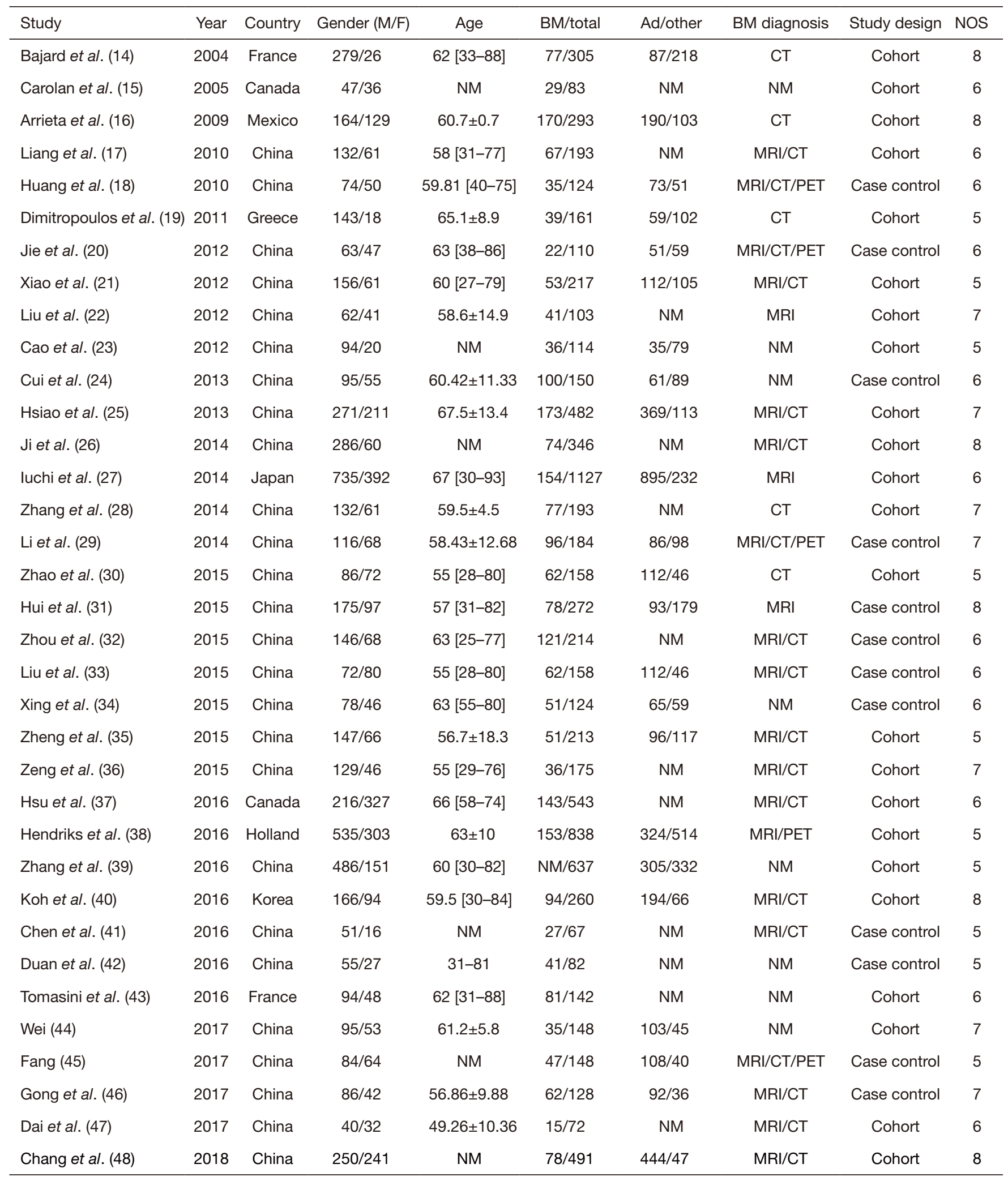

Table 1 (continued) 
Table 1 (continued)

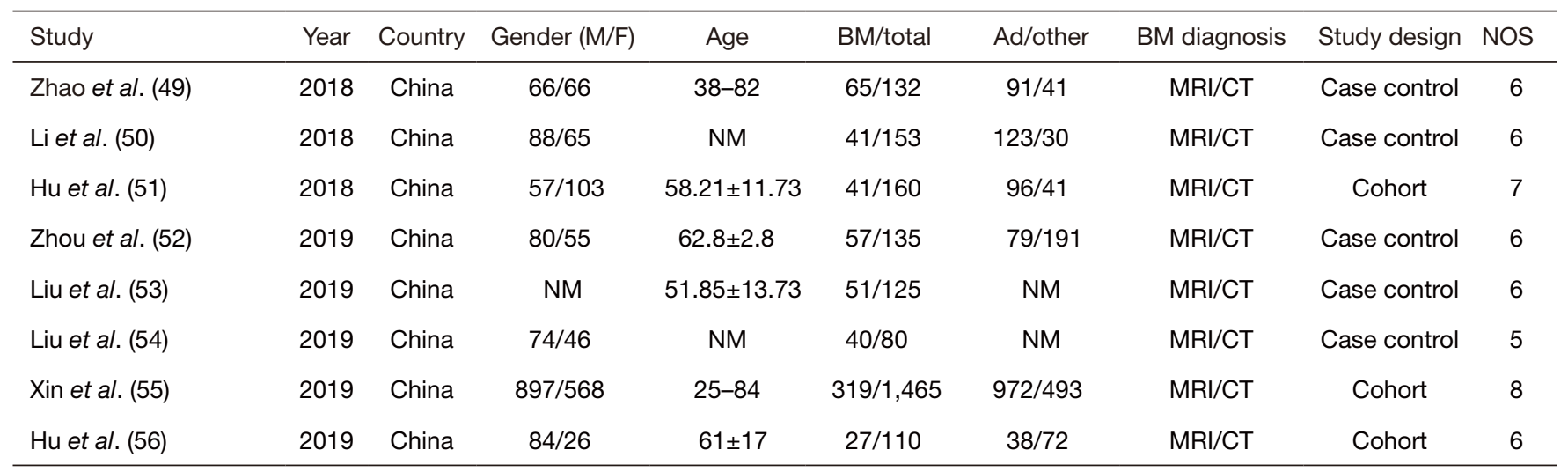

M/F, male/female; BM, brain metastasis; TNM, tumor node metastasis stage; Ad, adenocarcinoma; NOS, Newcastle Ottawa Quality Assessment Scale; MRI, magnetic resonance imaging; CT, computed tomography; PET, positron emission tomography; NM, not mentioned.

higher levels of CEA, CA199, CYFRA211, NSE, and CA125 were clinical risk factors that can predict BM.

Some multivariate analyses have already shown that the risk of $\mathrm{BM}$ is reduced with age; however, our meta-analysis found that age $\leq 60$ years old was associated with BM's incidence. The reason why an increased risk exists in the younger age cohort remains unclear; although, a possible mechanism for this may be due to the differential expression of some biological markers associated with BM, such as E-cadherin and Caspase-3, between younger and older patients (57). Also, it is well established that adenocarcinoma is common in females and often metastasizes to the brain, explaining why females have a higher incidence of BM (24). Moreover, a recent study (58) has demonstrated that the proportion of lung cancers diagnosed among smokers is increasing and that the risk of developing lung cancer is 20-40 times higher in smokers compared to never-smokers, which may explain the high proportion of smoking history in NSCLC patients with BM.

$\mathrm{BM}$ is closely associated with tumor-related indicators. In 2015, Won et al. (59) established a nomogram for predicting BM in NSCLC patients and found that histological type, T stage, and $\mathrm{N}$ stage were closely linked to BM. Similarly, Wang et al. (60) reported that non-squamous cell carcinoma and multiple lymphatic metastases were both risk factors for $\mathrm{BM}$, consistent with the results of our study. We found that non-squamous cell carcinoma, especially adenocarcinoma, was an independent risk factor for BM, which may be attributed to adenocarcinoma's invasive growth. Previous research (61) speculated that if a tumor spreads to the chest's lymphatic system, it will also involve distant metastasis to other organs (including bone, liver, and kidney), and if distant metastasis occurs, the probability of BM will increase. Our study is consistent with these studies in identifying lymphatic metastasis as a significant prognostic factor. A previous meta-analysis involving 22 studies reported that patients with EGFR mutation were more susceptible to BM than those with wild type EGFR (OR $=1.99,95 \%$ CI: 1.59-2.48, $\mathrm{P}=0.000)$ (62). A possible mechanism for this may involve EGFR activating MET via protein kinases and activating STAT3 via interleukin-6 to promote BM in NSCLC $(63,64)$.

Our study also found that the levels of relevant serum tumor markers were related to BM in NSCLC patients. We identified five prognostic factors: higher CEA levels, CA199, CYFRA211, NSE, and CA125. It has been previously reported that CEA-positive tumor cells can cross the blood-brain barrier more easily and adhere to the cerebral vasculature, which promotes the occurrence of BM (65). Our study also found that a higher level of CEA was a risk factor for BM. Meanwhile, previous studies $(45,49)$ demonstrated that serum CEA, CA199, CA125, and CYFRA211 were higher in the BM group than the control group, providing an important reference for the early detection of BM in NSCLC patients.

The results of the cohort and case-control studies were also analyzed separately (Table 2). The statistical results reported in the cohort studies were consistent with the results of the case-control studies, except for smoking history, adenocarcinoma, squamous cell carcinoma, and higher tumor stage. Considering that cohort studies had 


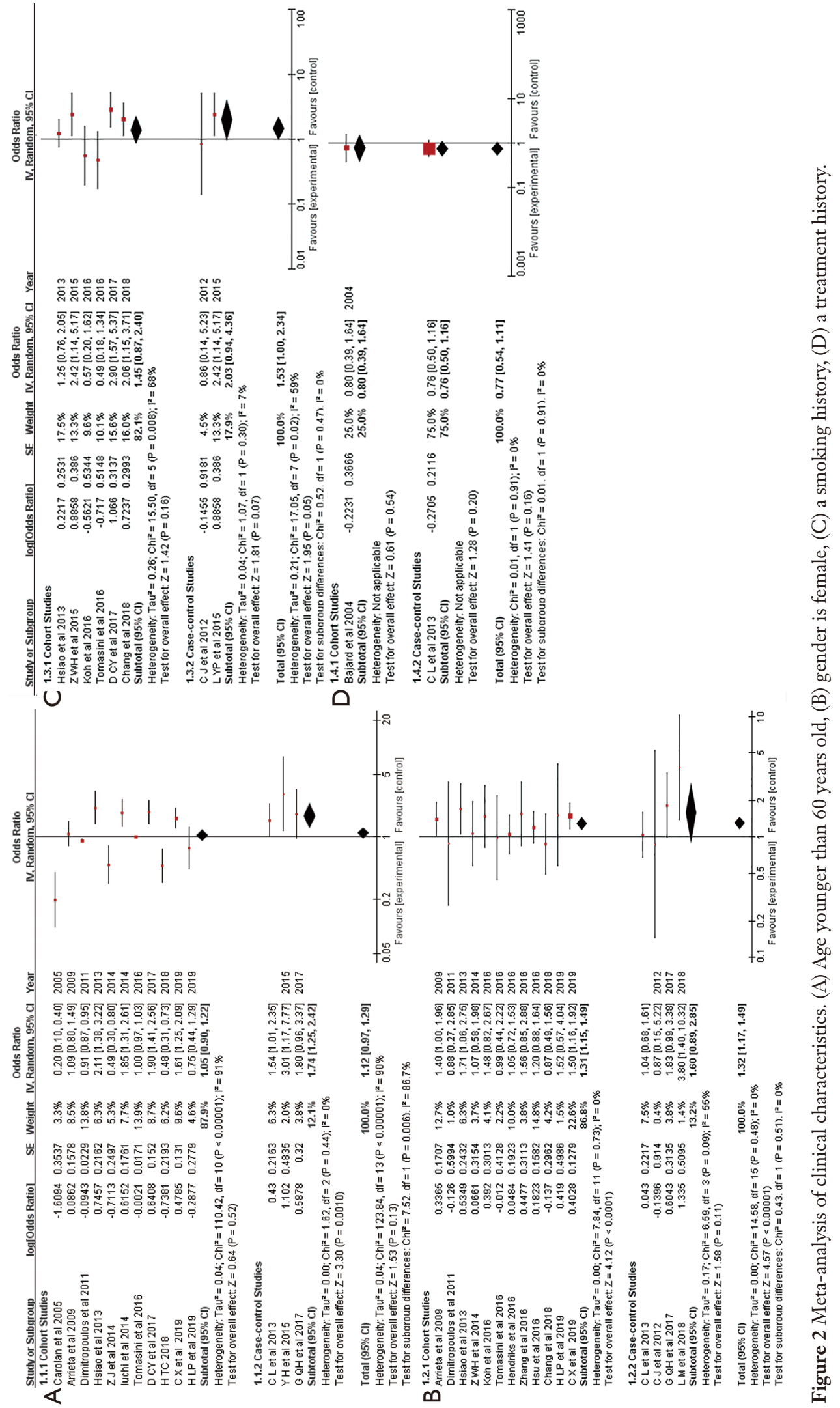




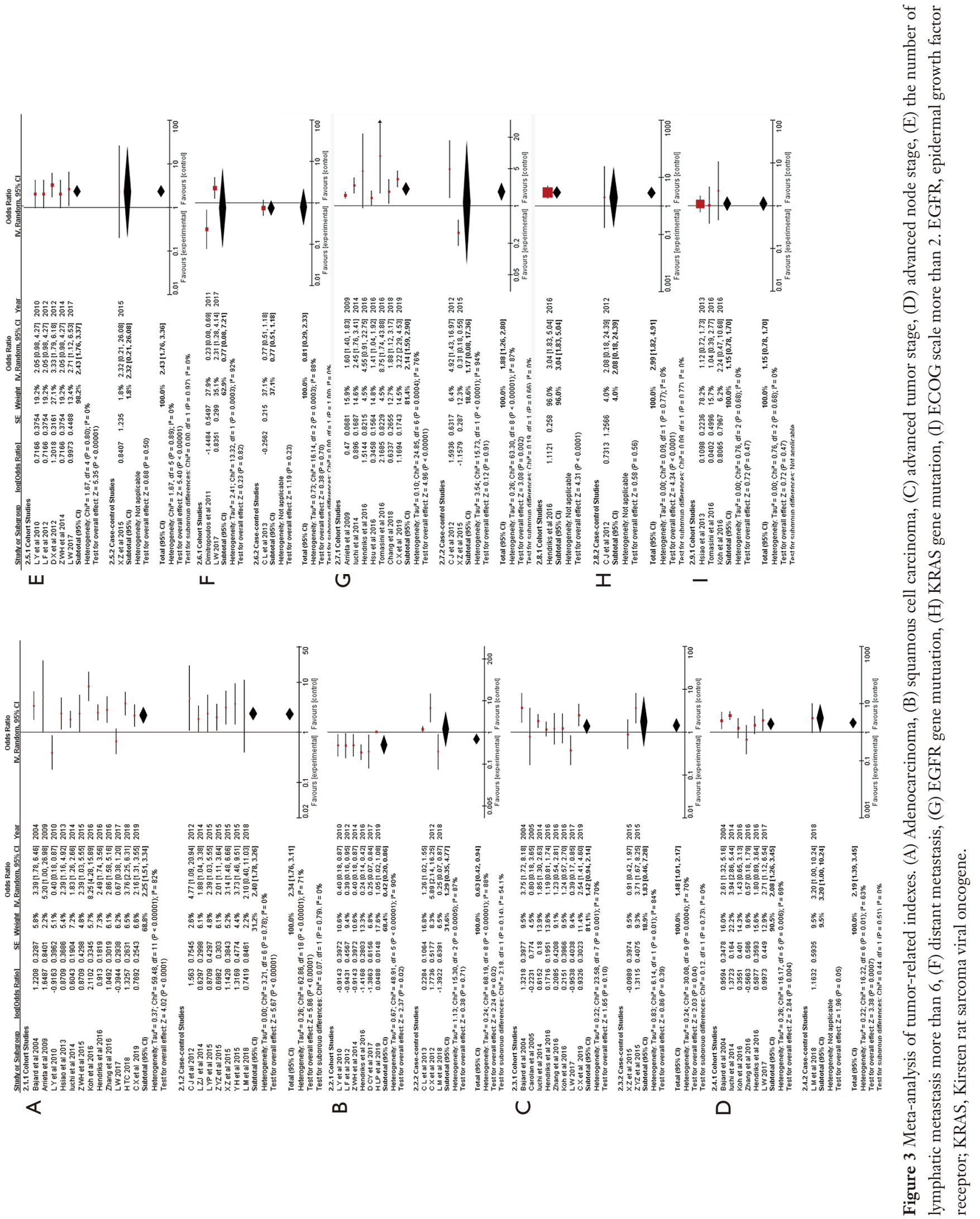




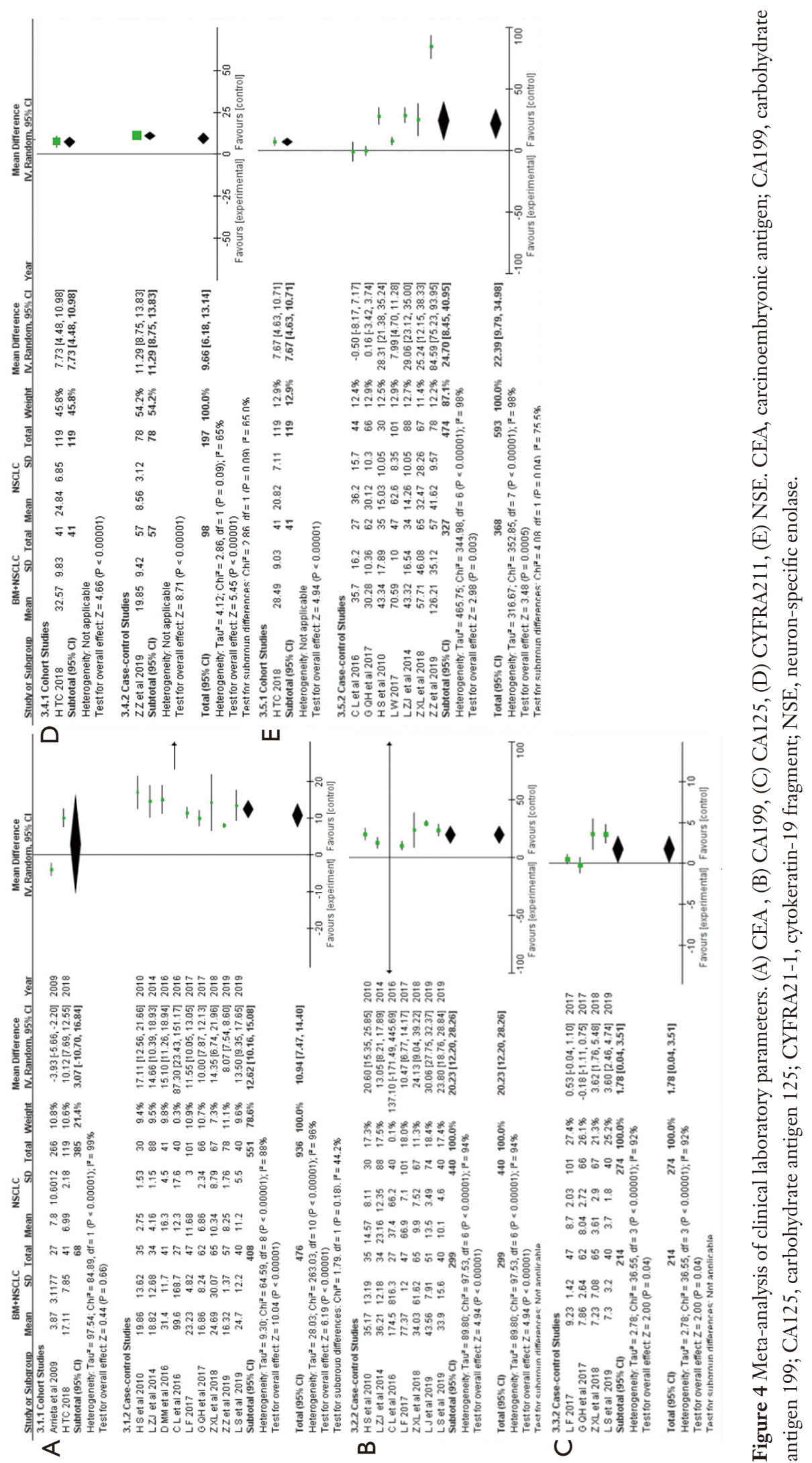



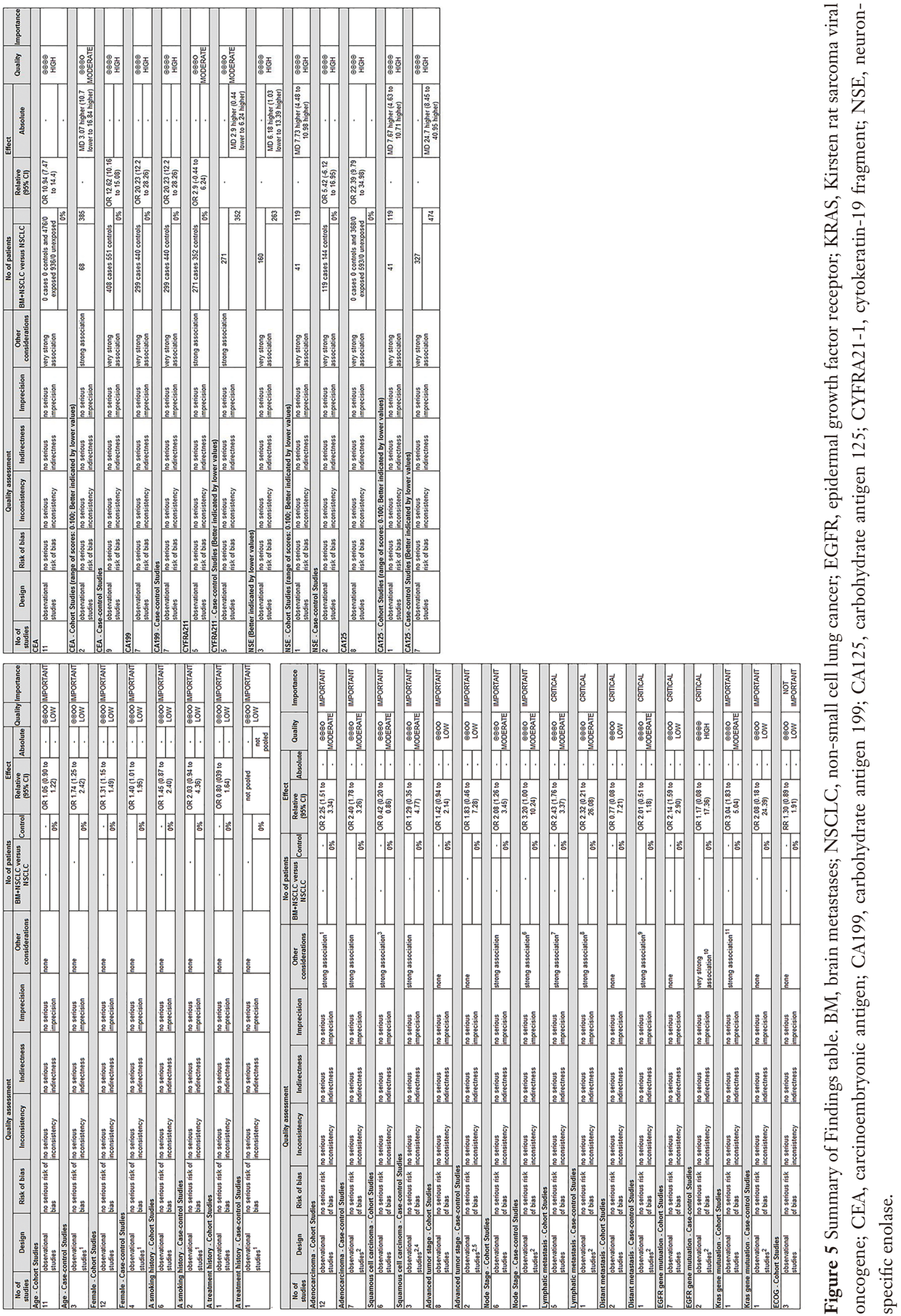

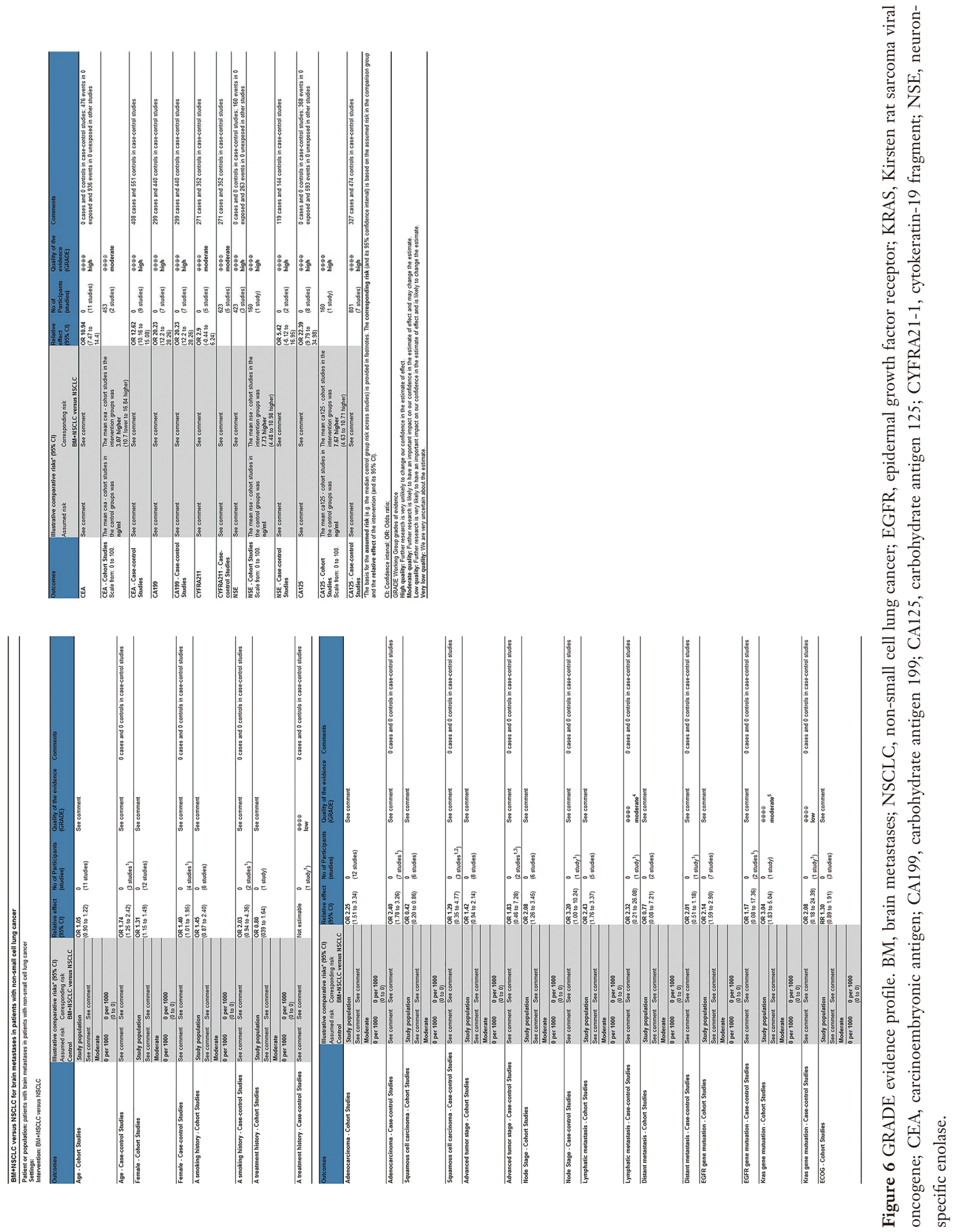
Table 2 The results stratified by study design for risk factors included in the meta-analysis

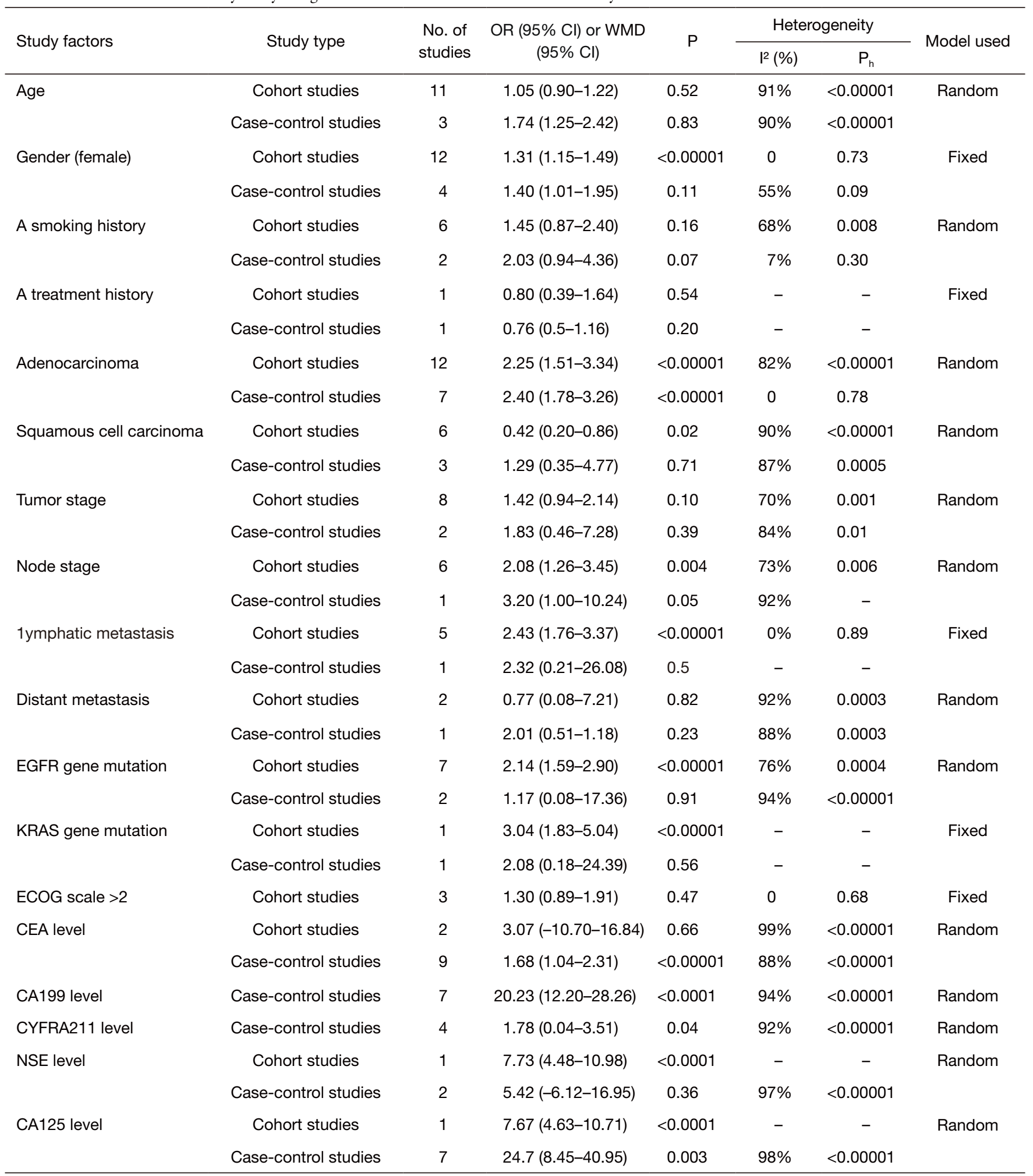

OR, odds ratio; WMD, weighted mean difference; $\mathrm{Cl}$, confidence interval; EGFR, epidermal growth factor receptor; KRAS, kirsten rat sarcoma viral oncogene; CEA, carcinoembryonic antigen; CA199, carbohydrate antigen 199; CYFRA21-1, cytokeratin-19 fragment; NSE, neuron-specific enolase; CA125, carbohydrate antigen 125. 
a higher proportion and were more likely to be authentic, we believe that random-effects models were suitable for these four indexes. Moreover, the heterogeneity of all five serum tumor markers was significant $(\mathrm{P}<0.05)$, which may be attributable to a failure to publish studies with negative results or different means of measurement. After stratifying by study design, we found that there were only case-control studies for some indexes, and thus, further investigation is required to confirm the conclusion.

Also, we assessed the differences in 1-, 2-, 3-, and 5 -year survival rates between NSCLC patients with BM and sampled NSCLC patients, respectively. The results indicated that the NSCLC + BM group had a significantly lower survival rate $(\mathrm{P}<0.05$, Figure $\mathrm{S} 6)$. A possible explanation for this is that patients with $\mathrm{BM}$ are more likely to present with distant metastasis of other sites, thus increasing these patients' mortality rates. Furthermore, the results also indicated that NSCLC patients with an advanced tumor stage were more likely to have BM, which can also decrease the survival time of NSCLC patients.

Our study had some shortcomings and omissions that should be noted. Firstly, the studies included in our metaanalysis were all either cohort or case-control studies, and the NOS quality assessment showed that the 43 included studies had relatively low scores (5-8), indicating that the results may have been subject to selection bias. Secondly, potential risk factors, such as cancer history, treatment approach, or other biological markers, could also promote BM's occurrence and affect the prognosis of cancers. However, these factors were not explored in this metaanalysis because the included studies may not have provided the required information. Thirdly, funnel plots showed no obvious publication bias for indexes with more than 10 relevant studies; however, potential bias could not be completely excluded for indexes with fewer than 10 studies.

\section{Conclusions}

In summary, our meta-analysis revealed that gender (female), adenocarcinoma or non-squamous cell carcinoma, advanced tumor stage, node stage, lymphatic metastasis, EGFR gene mutation, KRAS gene mutation, as well as higher levels of CEA, CA199, CYFRA211, NSE, and CA125 were risk factors for BM in NSCLC patients. We also determined that BM's presence could significantly decrease the survival time of NSCLC patients, indicating a poor survival prognosis. This meta-analysis demonstrated that NSCLC patients with $\mathrm{BM}$ have more aggressive clinical features and a poor survival prognosis.

\section{Acknowledgments}

Since the novel coronavirus spread throughout China and worldwide, all authors rushed to the forefront of the fight against the virus. Missions in the heart, responsibility in the shoulder. We extend our sincere gratitude to the families of all authors and teachers and colleagues for supporting us during this difficult time.

Funding: None.

\section{Footnote}

Reporting Checklist: The authors have completed the PRISMA reporting checklist. Available at http://dx.doi. org/10.21037/apm-20-1722

Conflicts of Interest: All authors have completed the ICMJE uniform disclosure form (available at http://dx.doi. org/10.21037/apm-20-1722). The authors have no conflicts of interest to declare.

Ethical Statement: The authors are accountable for all aspects of the work in ensuring that questions related to the accuracy or integrity of any part of the work are appropriately investigated and resolved.

Open Access Statement: This is an Open Access article distributed in accordance with the Creative Commons Attribution-NonCommercial-NoDerivs 4.0 International License (CC BY-NC-ND 4.0), which permits the noncommercial replication and distribution of the article with the strict proviso that no changes or edits are made and the original work is properly cited (including links to both the formal publication through the relevant DOI and the license). See: https://creativecommons.org/licenses/by-nc-nd/4.0/.

\section{References}

1. Torre LA, Siegel RL, Jemal A. Lung Cancer Statistics. In: Ahmad A, Gadgeel S, eds. Lung Cancer and Personalized Medicine: Current Knowledge and Therapies. Cham: Springer International Publishing 2016:1-19.

2. Li RY, Chen D. Analysis of Related Factors of Brain Metastasis in Lung Cancer. World Latest Medicine Information 2018;18:151-2.

3. Waqar SN, Samson PP, Robinson CG, et al. Non-small- 
cell Lung Cancer With Brain Metastasis at Presentation. Clin Lung Cancer 2018;19:e373-9.

4. Morgensztern D, Ng SH, Gao F, et al. Trends in stage distribution for patients with non-small cell lung cancer: a National Cancer Database survey. J Thorac Oncol 2010;5:29-33.

5. Ando T, Kage H, Saito M, et al. Early stage non-small cell lung cancer patients need brain imaging regardless of symptoms. Int J Clin Oncol 2018;23:641-6.

6. Petrovic M, Ilic N, Loncarevic O, et al. Risk factors for brain metastases in surgically staged IIIA non-small cell lung cancer patients treated with surgery, radiotherapy and chemotherapy. Vojnosanit Pregl 2011;68:643-9.

7. Xie SS, Li M, Zhou CC, et al. Prophylactic cranial irradiation may impose a detrimental effect on overall survival of patients with nonsmall cell lung cancer: a systematic review and meta-analysis. PLoS One 2014;9:e103431.

8. Cagney DN, Martin AM, Catalano PJ, et al. Incidence and prognosis of patients with brain metastases at diagnosis of systemic malignancy: a population-based study. Neuro Oncol 2017;19:1511-21.

9. Soffietti R, Kocher M, Abacioglu UM, et al. A European Organisation for Research and Treatment of Cancer phase III trial of adjuvant whole-brain radiotherapy versus observation in patients with one to three brain metastases from solid tumors after surgical resection or radiosurgery: quality-of-life results. J Clin Oncol 2013;31:65-72.

10. Stang A. Critical evaluation of the Newcastle-Ottawa scale for the assessment of the quality of nonrandomized studies in meta-analyses. Eur J Epidemiol 2010;25:603-5.

11. Guyatt GH, Oxman AD, Kunz R, et al. What is "quality of evidence" and why is it important to clinicians? BMJ 2008;336:995-8.

12. Asimit J, Day-Williams A, Zgaga L, et al. An evaluation of different meta-analysis approaches in the presence of allelic heterogeneity. Eur J Hum Genet 2012;20:709-712.

13. Li SJ, Huang J, Zhou XD, et al. Clinicopathological and prognostic significance of Oct-4 expression in patients with non-small cell lung cancer: a systematic review and meta-analysis. J Thorac Dis 2016;8:1587-600.

14. Bajard A, Westeel V, Dubiez A, et al. Multivariate analysis of factors predictive of brain metastases in localised nonsmall cell lung carcinoma. Lung Cancer 2004;45:317-23.

15. Carolan H, Sun AY, Bezjak A, et al. Does the incidence and outcome of brain metastases in locally advanced non-small cell lung cancer justify prophylactic cranial irradiation or early detection? Lung Cancer 2005;49:109-15.
16. Arrieta O, Saavedra-Perez D, Kuri R, et al. Brain metastasis development and poor survival associated with carcinoembryonic antigen (CEA) level in advanced nonsmall cell lung cancer: a prospective analysis. BMC Cancer 2009;9:119.

17. Liang Y, Zhang L, Wang S, et al. Clinical Predictive Factors for Brain Metastasis from Stage IIIA Non-SmallCell Lung Cancer after Surgery. Chinese Journal of Clinical Oncology 2010;37:1232-6.

18. Huang S, Qi YF. Study on Correlation between Non-small Cell Lung Cancer, Brain, Bone Metastasis and Tumor Markers. China: Shandong Traditional Chinese Medicine University 2010.

19. Dimitropoulos C, Hillas G, Nikolakopoulou S, et al. Prophylactic cranial irradiation in non-small cell lung cancer patients: who might be the candidates? Cancer Manag Res 2011;3:287-94.

20. Jie C. Evaluation of risk factors for brain metastasis and the effect of targeted therapy in non-small cell lung cancer patients with brain metastases. China: Qinghua Medical University 2012.

21. Xiao D. Risk factors of brain metastases in non-small cell lung cancer. China: Chinese Academy of Medical Sciences \& Peking Union Medical College 2012.

22. Liu F, Jia JH, Gong ZQ, et al. Analysis of related factors of brain metastasis in locally advanced non-small cell lung cancer. Modern Journal of Integrated Traditional Chinese and Western Medcine 2012;21:4018-9.

23. Cao X, Wang AL, Yang B, et al. Study on risk factors of brain metastases of locally advanced non-small-cell lung cancer. Cancer Research and Clinic 2012;24:24-7.

24. Cui L, Yan JH, Zhang JQ, et al. High risk factors and survival analysis of brain metastasis in non-small cell lung cancer. Journal of Practical Oncology 2013;28:45-8.

25. Hsiao SH, Chung CL, Chou YT, et al. Identification of subgroup patients with stage IIIB/IV non-small cell lung cancer at higher risk for brain metastases. Lung Cancer 2013;82:319-23.

26. Ji Z, Bi N, Wang J, et al. Risk factors for brain metastases in locally advanced non-small cell lung cancer with definitive chest radiation. Int J Radiat Oncol Biol Phys 2014;89:330-7.

27. Iuchi T, Shingyoji M, Itakura M, et al. Frequency of brain metastases in non-small-cell lung cancer, and their association with epidermal growth factor receptor mutations. Int J Clin Oncol 2015;20:674-9.

28. Zhang WH, Ye M, Zhou RW, et al. Analysis of clinical characteristics and risk factors of brain metastasis in stage 
IIIA non-small cell lung cancer after complete resection. Chinese Journal of Gerontology 2014;34:107-8.

29. Li Z, Li Z, Lu Q, et al. Clinical significance of combined detection of CA19-9, CA125, CEA and Ferritin in the diagnosis of NSCLC with brain or bone Metastasis. Progress in Modern Biomedicine 2014;14:3729-32.

30. Zhao WH, Liu YP, Yu QT, et al. Analysis of the relationship between serum CEA level and brain metastasis in patients with advanced non-small cell lung cancer. Journal of Guangxi Medical University 2015;32:401-4.

31. Hui Y. Relevant clinical and imaging predictors of metastatic disease to the brain from non-small cell lung carcinoma. China: Jinan University 2015.

32. Zhou JP, Wang L, Yang HX, et al. The risk factors of brain metastases in non-small cell Lung cancer patients after complete resection. Journal of Kunming Medical University 2015;36:138-41.

33. Liu YP. Risk factors for brain metastases in advanced nonsmall cell lung cancer. China: Guangxi Medical University 2015.

34. Xing Z, Wang $\mathrm{H}, \mathrm{Hu} \mathrm{Q}$, et al. Risk factors and survival analysis of locally advanced non-small cell lung cancer with brain metastasis. Journal of Clinical Medicine in Practice 2015;19:24-7.

35. Zheng YZ, Zhang X, Li H. Risk factor analysis for postoperative brain metastasis in patients with nonsmall-cell lung cancer. Journal of Medical Postgraduates 2015;28:953-956.

36. Zeng HY. Risk factors for brain metastases after prophylactic cranial irradiation in non-small cell cancer. China: Jinan Universtiy 2015.

37. Hsu F, De Caluwe A, Anderson D, et al. EGFR mutation status on brain metastases from non-small cell lung cancer. Lung Cancer 2016;96:101-7.

38. Hendriks LEL, Brouns JWM, Amini M, et al. Development of symptomatic brain metastases after chemoradiotherapy for stage III non-small cell lung cancer: Does the type of chemotherapy regimen matter? Lung Cancer 2016;101:68-75.

39. Zhang F, Zheng W, Ying L, et al. A Nomogram to Predict Brain Metastases of Resected Non-Small Cell Lung Cancer Patients. Ann Surg Oncol 2016;23:3033-9.

40. Koh YW, Choi JH, Ahn MS, et al. Baseline neutrophillymphocyte ratio is associated with baseline and subsequent presence of brain metastases in advanced nonsmall-cell lung cancer. Sci Rep 2016;6:38585.

41. Chen L, Zhang XL, Jiang F, et al. Diagnosis and prognosis of brain metastasis in lung cancer by tumor marker
D-dimer and platelet count. Zhejiang Clinical Medical Journal 2016;18:1011-3.

42. Duan MM, Fu JJ. Value of CA125, CEA, CA724 and P53 expression in diagnosis of NSCLC with brain metastases. Modern Hospital 2016;16:1611-3.

43. Tomasini P, Serdjebi C, Khobta N, et al. EGFR and KRAS Mutations Predict the Incidence and Outcome of Brain Metastases in Non-Small Cell Lung Cancer. Int J Mol Sci 2016;17:2132.

44. Wei L. Prediction effect of NSE to brain mestastasis in patients with non-small cell lung cancer before treatment and living condition. Medical Laboratory Science and Clinics 2017;28:12-5.

45. Fang L. Joint detection of serum tumor markers in the diagnosis and treatment of non-small cell lung cancer with brain metastases. The Practical Journal of Cancer 2017;32:578-81.

46. Gong QH, Liu YY, Gu XQ. Correlations analysis between serum tumor marker with clinical pathology, shortterm effect and prognosis of NSCLC patients with brain metastases. Med \& Pharm J Chin PLA 2017;29:31-5.

47. Dai CY, Wang LY, Yao DM, et al. Analysis of characteristics and risk factors of brain metastases following thoracic radiotherapy in surgery-ineligible patients with locally advanced non-small cell lung cancer. Oncology Progress 2017;15:312-33.

48. Chang WY, Wu YL, Su PL, et al. The impact of EGFR mutations on the incidence and survival of stages I to III NSCLC patients with subsequent brain metastasis. PLoS One 2018;13:e0192161.

49. Zhao XL, Kuang XK, Shi MY, et al. Application value of combined detection of four serum tumor markers in the diagnosis of brain metastasis of non-small cell lung cancer. Journal of Basic and Clinical Oncology 2018;31:57-9.

50. Li M, Li CL. Risk factors of brain metastasis in patients with non-small cell lung cancer. Journal of Logistics University of PAP (Medical Sciences) 2018;27:999-1002.

51. Hu T. Analysis of Risk Factors of Brain Metastasis in Patients with Advanced NSCLC. The Practical Journal of Cancer 2018;33:1885-9.

52. Zhou Z, Feng Q. Correlation between brain metastasis and serum tumor markers in patients with non-small cell lung cancer. Chinese Journal of Practical Nervous Diseases 2019;22:2097-103.

53. Liu J, Lv XC, Chen J, et al. A correlation analysis between brain metastases from lung cancer and serum NSE expression in patients. Chinese Journal of Surgical Oncology 2019;11:446-9. 
54. Liu S, Li XR. The clinical diagnostic value of joint detection of multiple tumor markers in the NSCLC patients with brain metastasis. Journal of North Sichuan Medical College 2019;34:548-50.

55. Xin C. Analysis of risk factors of brain metastases in advanced non-small cell lung cancer. China: China Medical University 2019.

56. Hu LP, Zou F, Fang CL, et al. Risk Analysis Brain Metastasis of Non-small Cell Lung Cancer (NSCLC) in Combination with Image Feature, Pathological Pattern and Ki-67 Gene Expression. The Practical Journal of Cancer 2019;34:219-21.

57. Saad AG, Yeap BY, Thunnissen FB, et al. Immunohistochemical markers associated with brain metastases in patients with nonsmall cell lung carcinoma. Cancer 2008;113:2129-38.

58. Chen YX, Guo Y, Dong SS, et al. Runs of homozygosity associate with decreased risks of lung cancer in neversmoking East Asian females. J Cancer 2018;9:3858-66.

59. Won YW, Joo J, Yun T, et al. A nomogram to predict brain metastasis as the first relapse in curatively resected nonsmall cell lung cancer patients. Lung Cancer 2015;88:201-7.

60. Wang SY, Ye X, Ou W, et al. Risk of cerebral metastases

Cite this article as: Chen S, Hua X, Jia J, Wu Y, Wei S, Xu L, Han S, Zhang H, Zhu X. Risk factors for brain metastases in patients with non-small cell lung cancer: a meta-analysis of 43 studies. Ann Palliat Med 2021;10(4):3657-3672. doi: 10.21037/apm20-1722 for postoperative locally advanced non-small-cell lung cancer. Lung Cancer 2009;64:238-43.

61. Mujoomdar A, Austin JH, Malhotra R, et al. Clinical predictors of metastatic disease to the brain from nonsmall cell lung carcinoma: primary tumor size, cell type, and lymph node metastases. Radiology 2007;242:882-8.

62. Li L, Luo S, Lin H, et al. Correlation between EGFR mutation status and the incidence of brain metastases in patients with non-small cell lung cancer. J Thorac Dis 2017;9:2510-20.

63. Breindel JL, Haskins JW, Cowell EP, et al. EGF receptor activates MET through MAPK to enhance non-small cell lung carcinoma invasion and brain metastasis. Cancer Res 2013;73:5053-65.

64. Gao SP, Mark KG, Leslie K, et al. Mutations in the EGFR kinase domain mediate STAT3 activation via IL-6 production in human lung adenocarcinomas. J Clin Investig 2007;117:3846-56.

65. Liu ZJ, Shi Q, Lu B, Zhang Z, et al. Conventional Tumor Markers in Cerebralspinal Fluid in Patients with Elevated Serum Tumor Markers and without Central Nervous System Malignant Diseases. Zhongguo Yi Xue Ke Xue Yuan Xue Bao 2016;38:539-42. 


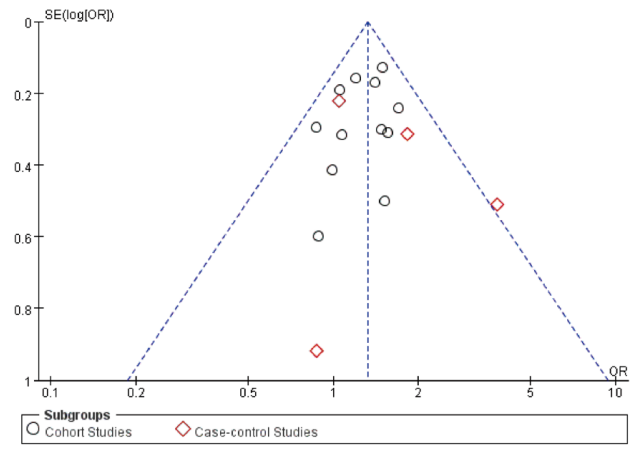

Figure S1 Funnel plots for the indexes (Female).

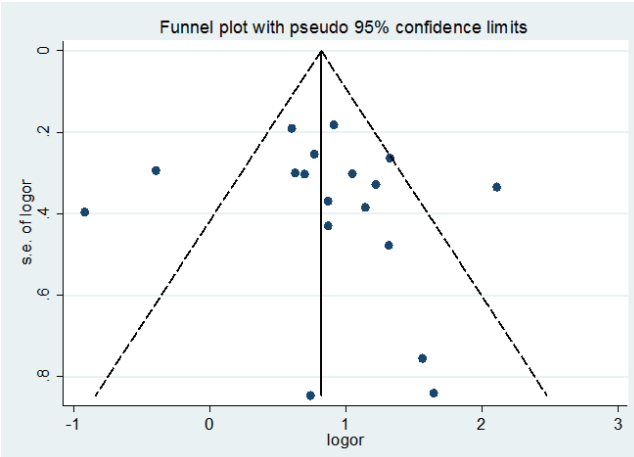

Figure S3 Funnel plots for the indexes (adenocarcinoma).

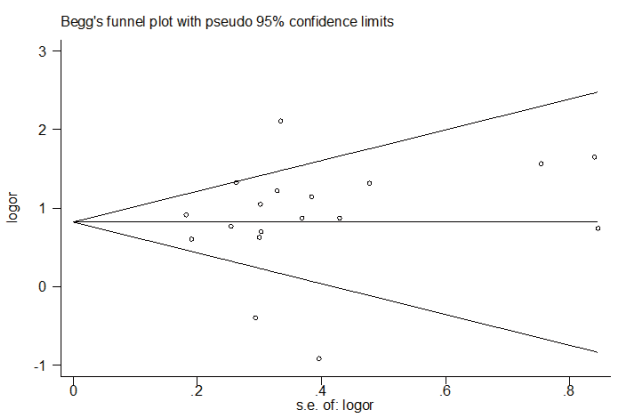

Figure S5 Begg's funnel plot (adenocarcinoma).

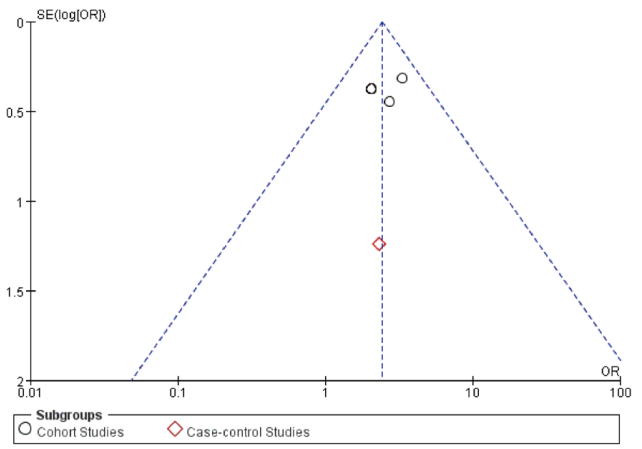

Figure S2 Funnel plots for the indexes (1ymphatic metastasis larger than 6).

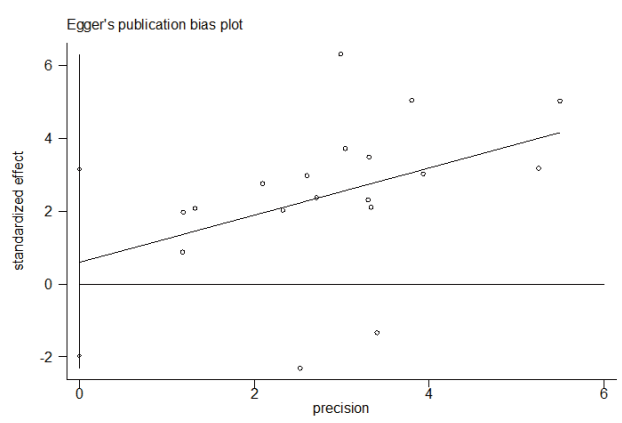

Figure S4 Egger's publication bias plot (adenocarcinoma). 


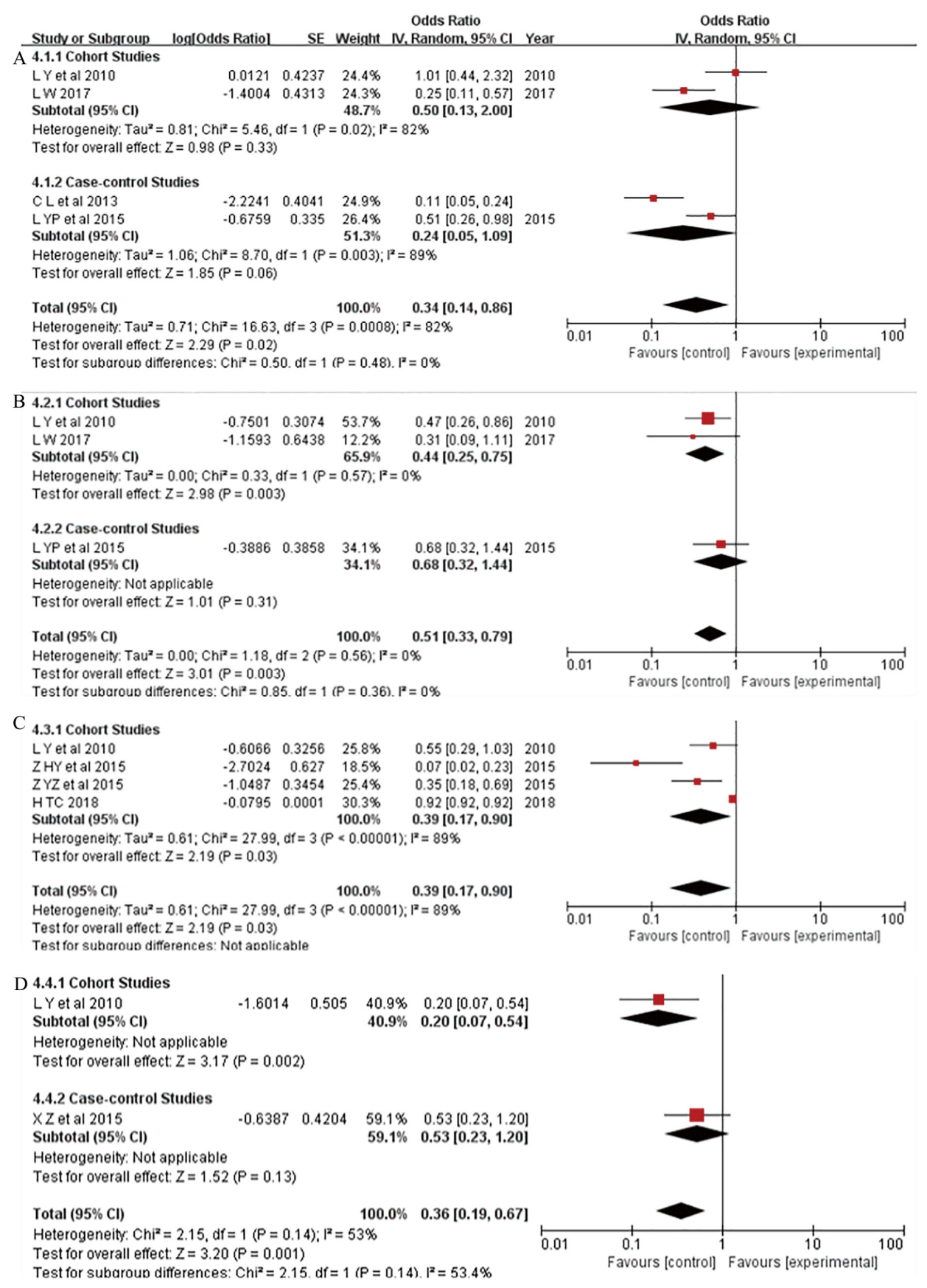

Figure S6 Meta-analysis of survival rates. (A) 1-year survival rate; (B) 2-year survival rate; (C) 3-year survival rate; (D) 5-year survival rate. 